\title{
Construction of fuzzy edge image using Interval Type II fuzzy set
}

\author{
Tamalika Chaira* \\ Centre for Biomedical Engineering, Indian Institute of Technology Delhi, New Delhi, INDIA
}

\author{
A. K. Ray \\ Department of Electronics and Electrical Comm. Engg., Indian Institute of Technology Kharagpur and Vice Chancellar \\ of Bengal Engg. And Science University, Sibpur, Howrah, INDIA
}

Received 17 January 2013

Accepted 29 April 2013

\begin{abstract}
In this paper, a novel method to generate fuzzy edges in medical images using the Type II fuzzy set theory is presented. Medical images are normally poorly illuminated and many edges are not visible properly, so construction of fuzzy edge image is a difficult task. Fuzzy edges are not the binary edges but it signifies the change in intensity levels of the image. The method is based on computation of minimum and maximum values of the intensity levels of the image in a $3 \times 3$ pixel neighborhood to form two image matrices with maximum and minimum values. For better representation of uncertainty, Type II fuzzy set is applied to compute upper and lower membership levels of each image matrix. Divergence is computed between the two levels of the maximum value image matrix and also for the minimum value image matrix. Finally, difference between the divergence matrices produces an edge image. Experiment has been performed on several poorly illuminated medical images and the edges are observed to better when compared with the existing edge methods.
\end{abstract}

Keywords: Type II fuzzy set, fuzzy T norm, fuzzy T co norm, fuzzy divergence, medical image

\section{Introduction}

Zadeh $^{1}$ introduced the concept of fuzzy set theory for modeling the vague concepts in the real world. In fuzzy set theory, elements partially belong to a set and their degree of membership is computed in terms of the membership function. In image, membership function denotes returns the degree of membership of the pixels in an image. But, there may be another degree of freedom in order to have more information in representing ordinary fuzzy set. The membership function of ordinary or Type I fuzzy set is considered fuzzy and so Zadeh ${ }^{2}$ in 1975 introduced Type II fuzzy set. Type II fuzzy set does not have precise membership value and is characterized as fuzzy but the membership function in Type I fuzzy set is crisp. Recently Type II fuzzy set has been an attracting interest in image processing fields. Some work on image thresholding using Type II fuzzy $\operatorname{set}^{3,4}$ are in literature. In their work, the images are thresholded as object and background. If medical images are dealt, abnormal

\footnotetext{
*Corresponding author: tchaira@yahoo.com.
} 
lesions or tumor can be detected using thresholding method.

There is also little work on edge detection. Edge in an image is defined as the boundary or the contour of the image and it occurs when there is a change in gray levels. They are the high frequency components of the image. Many authors suggested different edge detection techniques. Edge image gives the outline of the image object or structures that are useful in further analysis and recognition. There are many different methods for edge detection. Among them, the traditional methods are Sobel, Prewitt operators, Laplacian of Gaussian ${ }^{5}$. Canny ${ }^{6}$ approach is a classical method used for edge detection. Russo ${ }^{7}$ and Russo and Ramponi ${ }^{8}$ suggested fuzzy method for edge detection. Liang ${ }^{9}$ had given the idea for fuzzy edge detection that is computational faster than Canny's method. These methods smooth the images but use too many fuzzy rules. Chaira and Ray ${ }^{10}$ suggested an edge detection method using intuitionistic fuzzy set. Here 16 edge detection templates are used to detect the edges in different direction. Barrenchea et al. ${ }^{11}$ suggested interval valued fuzzy relation to construct fuzzy edges and Bustince et al. ${ }^{12}$ constructed edges using interval valued sets. In Barrenchea's method, they constructed interval valued fuzzy relation using lower constructor that uses $\mathrm{t}$ norm (minimum value) and upper constructor that uses $t$ co-norm (maximum value) to measure the intensity variation of a pixel and its neighborhood,. Fuzzy edge is generated using upper and lower constructor and the length of the interval denotes the intensity variation of a pixel in its neighborhood. Fuzzy edges can also be obtained using fuzzy mathematical morphology ${ }^{13}$ by Alper PAHSA, They used Sakawa and Yumine's fuzzy membership function to fuzzify the image. Structuring element is also fuzzified. In fuzzy mathematical morphology, maximum and minimum operators are replaced by $s$ and $\mathrm{t}$ norms. Then supremum and infimum are replaced by argmax and argmin. Fuzzy structuring element is used. Edge image is obtained by taking the difference between the dilated and eroded images.

In this paper, we propose a method to characterize fuzzy edge that should represent variations in intensity. An idea is developed to generate fuzzy edges that use Type II fuzzy set. Initially maximum and minimum intensity levels of the image are formed for a pixel in its $3 \times 3$ neighborhood to obtain two matrices. One point is to be noted, if we had taken the difference between the two matrices (maximum and minimum), fuzzy edges are not bright. For this, we computed upper and lower membership levels of each image matrix using Type II fuzzy set. Divergence between the two levels of the maximum value image matrix and that of minimum value image matrix are computed. Finally difference between the two divergence matrices produces a fuzzy edge image. Fuzzy edge is not an edge image like Canny ${ }^{4}$ and other edge detectors that produce binary image rather it captures the intensity change in an image.

The remainder of the paper is organized as follows. Section 2 introduces the theory of Type II fuzzy set. Section 3 details the methodology. Section 4 exhibits and discusses the experimental results. Lastly, section 5 provides conclusions of this paper.

\section{Introduction to Type II Fuzzy Set}

Type II fuzzy set is useful in situation where the membership values are inaccurate and is difficult to agree with the accurate membership values. Because, there may be some uncertainty in the location, or shape or other parameters ${ }^{14,15}$, Type II fuzzy set consists of another degree of freedom for better representation of uncertainty where the membership functions are themselves considered to be fuzzy. If there is no uncertainty, then a type-II fuzzy set reduces to a type-1 fuzzy set. A Type II fuzzy set is written as:

$$
A_{\text {TypeII }}=\left\{x, \widehat{\mu}_{A}(x) \mid x \in X\right\}
$$

where $\widehat{\mu}_{A}(x)$ type-II membership function. The footprint of uncertainty (FOU), represents the uncertainty in the primary memberships of the type-II fuzzy set as shown in Fig. $1^{16}$.

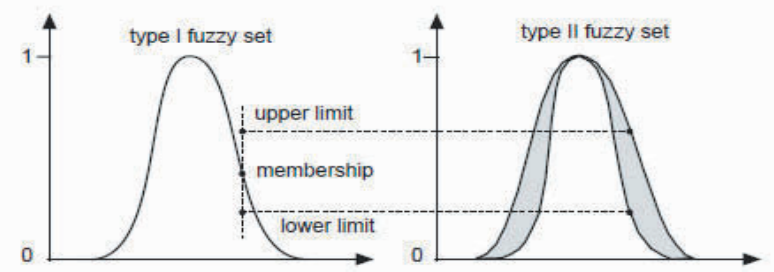

(a)

(b)

Fig.1. a) Type I membership function, b) interval of Type II fuzzy membership function (shaded region) is the FOU

Lower and upper membership function constitutes interval-valued fuzzy set and this is a special case of Type II fuzzy set. The upper and lower membership functions are written as:

$$
\begin{aligned}
& \mu^{\text {upper }}=[\mu(x)]^{\alpha} \\
& \mu^{\text {lower }}=[\mu(x)]^{1 / \alpha}
\end{aligned}
$$


where $\alpha \in[0,1]$. So, a more practical form of

representing Type II fuzzy set is:

$A_{\text {TypeII }}=\left\{x, \mu_{U}(x), \mu_{L}(x) \mid x \in X\right\}$

and $\mu_{U}(x)<\mu(x)<\mu_{L}(x), \mu \in[0,1]$

\section{The proposed technique}

Consider an image $\mathrm{A}$ and is normalized using the formula:

$\mu_{A}(g)=\frac{g_{i j}-g_{\text {min }}}{g_{\text {max }}-g_{\text {min }}}$

where $g_{i j}$ is the pixel value at $(i, j)^{t h}$ position. $g_{\text {min }}$, $g_{\max }$ are the minimum and maximum values of the gray levels of the image. This has been done to have the values in the interval $[0,1]$.

For each pixel, a $3 \times 3$ neighborhood is selected and the minimum and maximum values are noted. This is done for all the pixels. In this way, maximum membership value $\left(\mu_{\max }(g)\right)$ and minimum membership value $\left(\mu_{\min }(g)\right)$ are obtained. Thus we obtain two image matrices that correspond to brighter and darker gray levels of the image respectively.

Now for the maximum image membership matrix, upper and lower membership levels are computed using Type II fuzzy set theory. Likewise for the minimum image membership matrix, upper and lower membership levels are computed.

It is to be noted that maximum and minimum valued image matrices are not used for building Type II fuzzy set. Considering the image to be fuzzy, the maximum and minimum valued matrices are also considered fuzzy. For better representation of uncertainty, Type II fuzzy set is used to find the upper and lower membership levels of the two minimum and maximum matrices.

The upper and lower levels of the maximum membership values (from eq. (1)) are written as:

$$
\begin{aligned}
& \mu_{\max }^{\text {upper }}(g)=\left[\mu_{\max }(g)\right]^{0.75} \\
& \mu_{\max }^{\text {lower }}(g)=\left[\mu_{\max }(g)\right]^{1 / 0.75}
\end{aligned}
$$

$\alpha=0.75$ is chosen. After thorough experimentation it is found that $0.5<\alpha<1$ gives better result in terms of fuzzy edges. A sample result is shown in Fig. 2 for $\alpha=0.3$ and $\alpha=0.75$ where it is observed that with $\alpha=0.3$, the edges are little bit lighter.
Fuzzy divergence or the difference is computed between the upper and lower membership levels of the maximum valued image matrix. This is calculated as follows:

$$
\begin{aligned}
& \operatorname{Div}^{\max }\left(\mu_{\max }^{\text {upper }}(g), \mu_{\max }^{\text {lower }}(g)\right)=2-\left(1-\mu_{\max }^{\text {upper }}(g)+\right. \\
& \left.\mu_{\max }^{\text {lower }}(g)\right) \cdot e^{\mu_{\min }^{\text {upper }}(g)-\mu_{\min }^{\text {lower }}(g)}- \\
& \left(1-\mu_{\max }^{\text {lower }}(g)+\mu_{\max }^{\text {upper }}(g)\right) \cdot e^{\mu_{\min }^{\text {lower }}(g)-\mu_{\min }^{\text {upper }}(g)}
\end{aligned}
$$

Likewise, upper and lower levels of the minimum valued image matrix are also computed as eq. (2).

$$
\begin{aligned}
& \mu_{\min }^{\text {upper }}(g)=\left[\mu_{\min }(g)\right]^{0.75} \\
& \mu_{\min }^{\text {lower }}(g)=\left[\mu_{\min }(g)\right]^{1 / 0.75}
\end{aligned}
$$

And the divergence between the two membership levels of the minimum value image matrix which is given as:

$$
\begin{aligned}
& \operatorname{Div}^{\min }\left(\mu_{\min }^{\text {upper }}(g), \mu_{\min }^{\text {lower }}(g)\right)=2-\left(1-\mu_{\min }^{\text {upper }}(g)+\right. \\
& \left.\mu_{\min }^{\text {lower }}(g)\right) \cdot e^{\mu_{\min }^{\text {upper }}(g)-\mu_{\min }^{\text {lower }}(g)}- \\
& \left(1-\mu_{\min }^{\text {lower }}(g)+\mu_{\min }^{\text {upper }}(g)\right) \cdot e^{\mu_{\min }^{\text {lower }}(g)-\mu_{\min }^{\text {upper }}(g)}
\end{aligned}
$$

So, two divergence matrices are formed that is minimum divergence and maximum divergence. Then the difference between the two divergence matrices are computed using eqs. 3-4. The difference matrix so formed is an edge image.

Fuzzy Edge_image $=($ Div_max - Div_min $)$.

Doing this, each pixel is associated with different membership degree corresponding to its interval length (divergence between the maximum and minimum matrices).

In fuzzy morphology, difference between the dilation and erosion is computed using $\mathrm{s}$ and $\mathrm{t}$ norms to obtain an edge image. Fuzzy structuring element is used. Here also, some kind of difference between the maximum and minimum image value is taken.

The algorithm of the proposed scheme is given.

\section{The Algorithm:}

1. Select an image and normalize it.

2. For each pixel, compute minimum value in a $3 \times 3$ neighborhood and form a matrix. 
3. Likewise for each pixel compute the maximum value of the $3 \times 3$ neighborhood and form a matrix.

4. For each matrix - minimum and maximum valued matrices, calculate upper and lower membership levels using Type II fuzzy set using eq. (2).

5. Compute the divergence between the upper and lower membership levels of the maximum matrix to form a divergence_max matrix.

6. Likewise, compute the divergence between the upper and lower membership levels of the minimum matrix to form a divergence_min matrix.

7. Take the difference of the two divergence matrices to obtain an edge image.

\section{Results and Discussion}

Experiments have been performed on several images and the results on eight images are shown. We do not focus to develop an edge detector to produce binary edges rather we focus on to develop fuzzy edge. Results have been compared with fuzzy edges using fuzzy morphology ${ }^{13}$, interval valued fuzzy relation based edge images ${ }^{11}$, and the edge image (not the binary image) of Chaira and Ray ${ }^{9}$. For comparison, hand drawn images are considered as ground truth image. But medical images are poorly illuminated and so many of our image edges are not visible clearly thereby it becomes very difficult to draw. Visually, the edge images using our method are brighter and better than the existing methods. So, in this paper, visual comparison is done.

Fig. 3 is an image of knee patella. Result using fuzzy morphology in Fig 3(b) and edge image using Chaira in Fig. 3(d) show very vague edges where the difference between the dilated and eroded images is taken. Fuzzy edges using interval valued fuzzy relation in Fig. 3(c) shows that the knee bone and patella edges are somehow visible where as the result using the proposed method in Fig. 3(e) shows clear patella and knee bones. Fig. 4 is an image of blood cell, which is itself not bright. Edges using fuzzy morphology in Fig. 4(b) are not visible properly. Chaira and Ray's method in Fig. 4(d) does not show an edge image. Fuzzy edges using interval valued fuzzy set in Fig. 4(c) shows edges that are little bit visible where as the result using the proposed method in Fig. 4(e) shows clear edges of blood cells.

Fig 5 is an image of cell image. Edges using fuzzy morphology and Chaira and Ray in Figs. 5(b)-(c) are not clear and the edges using interval valued fuzzy set Fig. 5 (c) are little bit clear than that of Fig, 5(b) but the edges using the proposed method in Fig. 5(e) is much clear and better.

Figs $6,7,8$ are the images of cell image. Edges using fuzzy morphology in Figs. 6-8(b) are not visible properly. Edge image using Chaira and Ray in Figs. 6$8(d)$ are little bit better than that of morphology where edge are somewhat visible. Result using interval valued fuzzy set in Fig. 6-8 (c) shows unclear edges in the middle part of the image but the edges using the proposed method in Fig. 6-8(e) is visible clearly in the overall image.

Fig. 9 is an image of nuclei. Edge image using fuzzy morphology and Chaira and Ray in Figs. 9(b)-(c) are not at all clear. Edges using interval valued fuzzy set in Fig. 9 (c) are little bit visible where as the edges using the proposed method in Fig. 10(d) are bright with clear boundaries.

Fig. 10 is a common Lena image. Edge image using fuzzy morphology and Chaira and Ray in Figs. 10(b)-(c) are not clear. Edges using interval valued fuzzy set in Fig. 10 (c) are little bit visible where as the edges using the proposed method in Fig. 10(d) are bright with clear boundaries.

The idea behind the method is that instead of finding the intensity image directly using the maximum (brighter image) and minimum (darker image) image matrices (as in Barrenchea et at. ${ }^{9}$ ), upper and lower membership levels of each image matrix (maximum and minimum image matrices) are computed using Type II fuzzy set where additional degree of freedom is considered. This is applied to the systems where lots of uncertainties are present. As medical images contain lot of uncertainties, Type II fuzzy set is applied to the two image matrices to find the upper and lower membership functions. Divergence is computed between the two levels of the maximum image matrix and minimum image matrices separately. Doing this, each pixel is associated with interval length corresponding to the maximum and minimum values of the divergence of the two image matrices. So, the edge image using the proposed method is brighter than the method suggested by Barrenchea et al. ${ }^{9}$ that uses interval valued fuzzy relation i.e. a some kind of difference between the maximum and minimum value ( $\mathrm{t}$ norm and $\mathrm{t}$ co norm). 




(a)

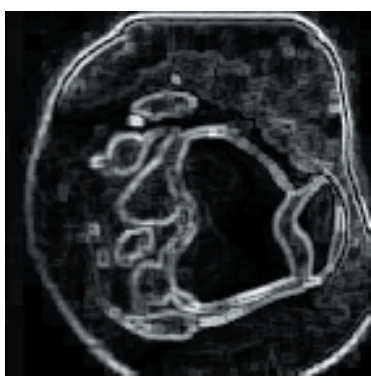

(c)

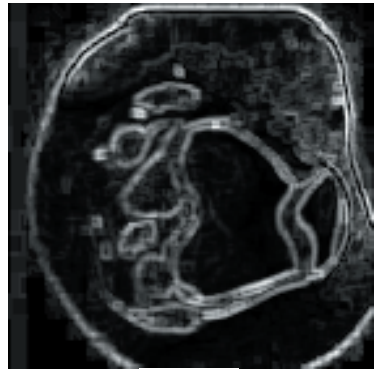

(b)
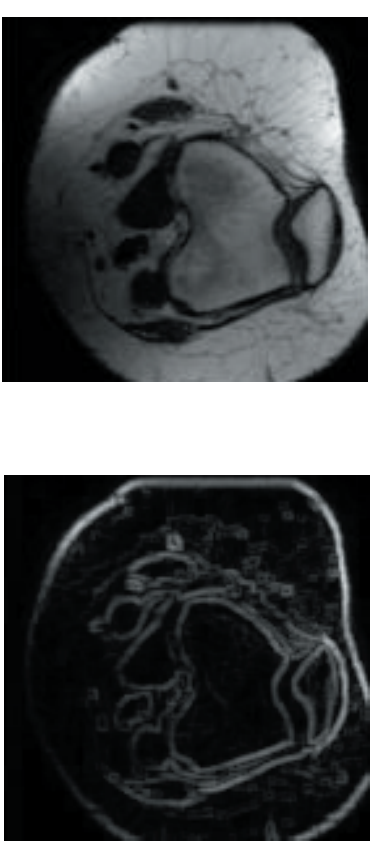

(c)

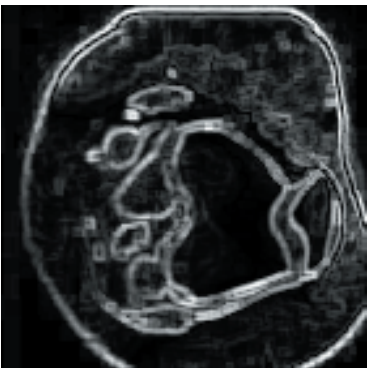

(e)

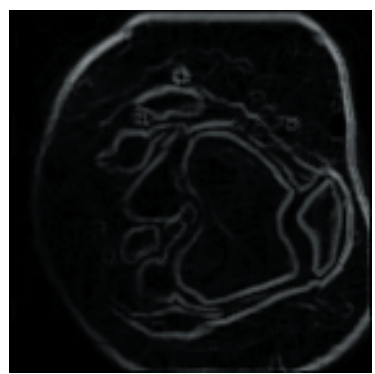

(b)



(d)

Fig. 2. : a) knee patella image, b) edge image using $\alpha=0.3$, c) edge image using $\alpha=0.75$

Fig. 3. : a) knee patella image, b) edge image using fuzzy morphology, c) edge image using the interval valued fuzzy set, d) edge image by Chaira and Ray, e) edge image using proposed method 


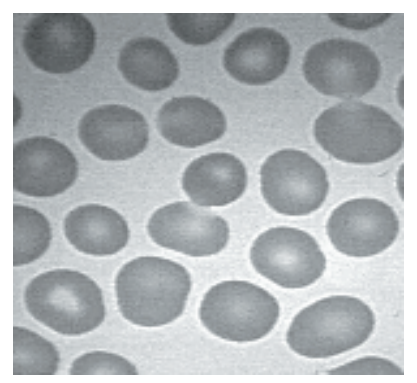

(a)

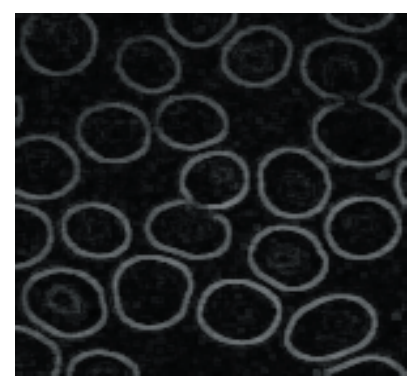

(c)

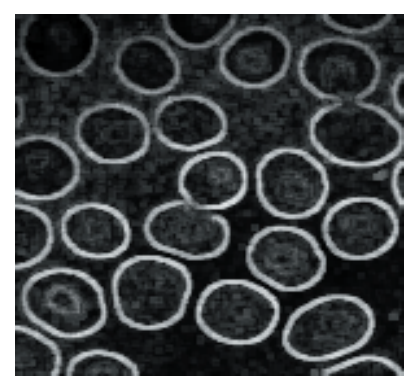

(e)

Fig. 4. : a) blood cell image, b) edge image using fuzzy morphology, c) edge image using the interval valued fuzzy set, d) edge image by Chaira and Ray, e) edge image using proposed method

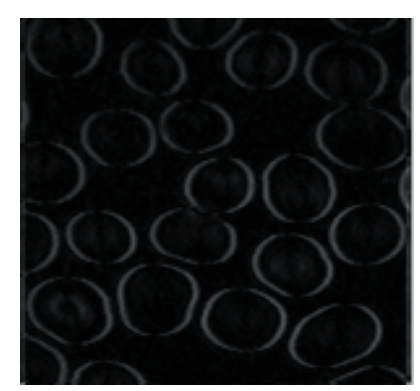

(b)

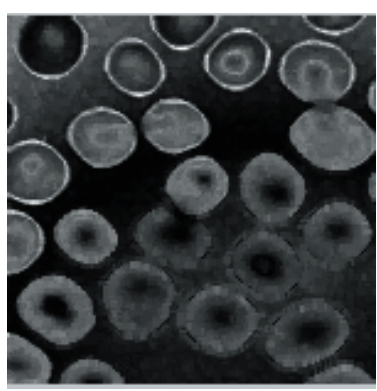

(d)

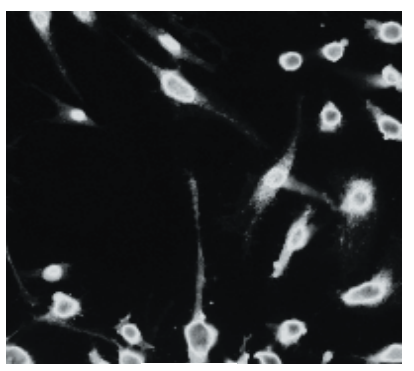

(a)

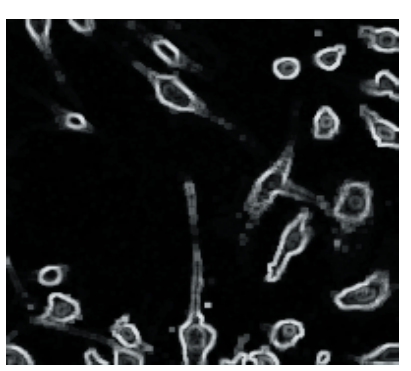

(c)

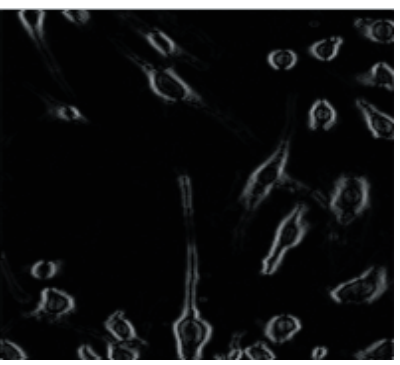

(b)

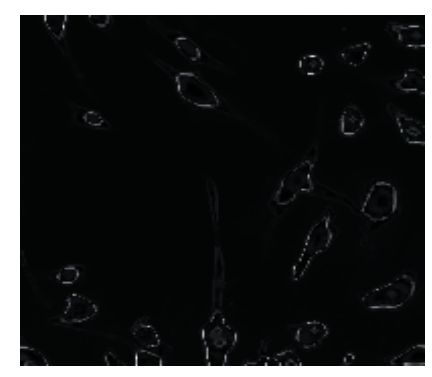

(d)



(e)

Fig. 5. : a) cell image, b) edge image using fuzzy morphology, c) edge image using the interval valued fuzzy set, d) edge image by Chaira and Ray, e) edge image using proposed method 


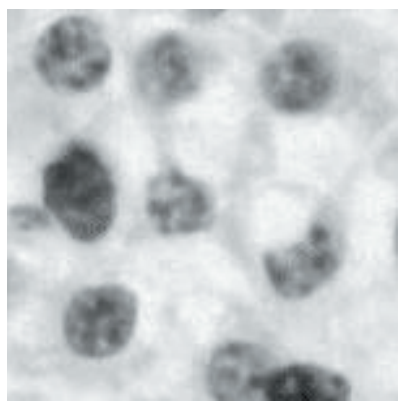

(a)

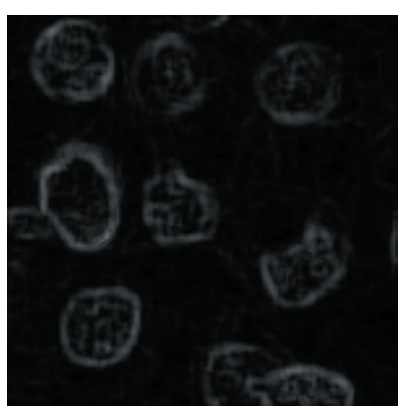

(c)

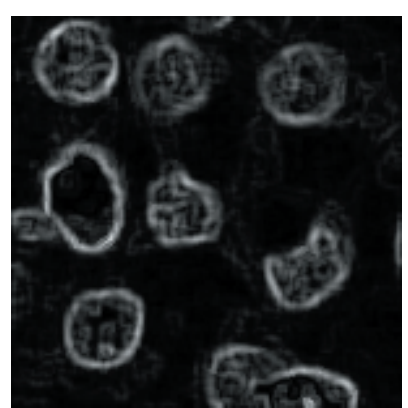

(e)

Fig. 6. : a) blood cell image, b) edge image using fuzzy morphology, c) edge image using the interval valued fuzzy set, d) edge image by Chaira and Ray, e) edge image using proposed method

(b)

(d)
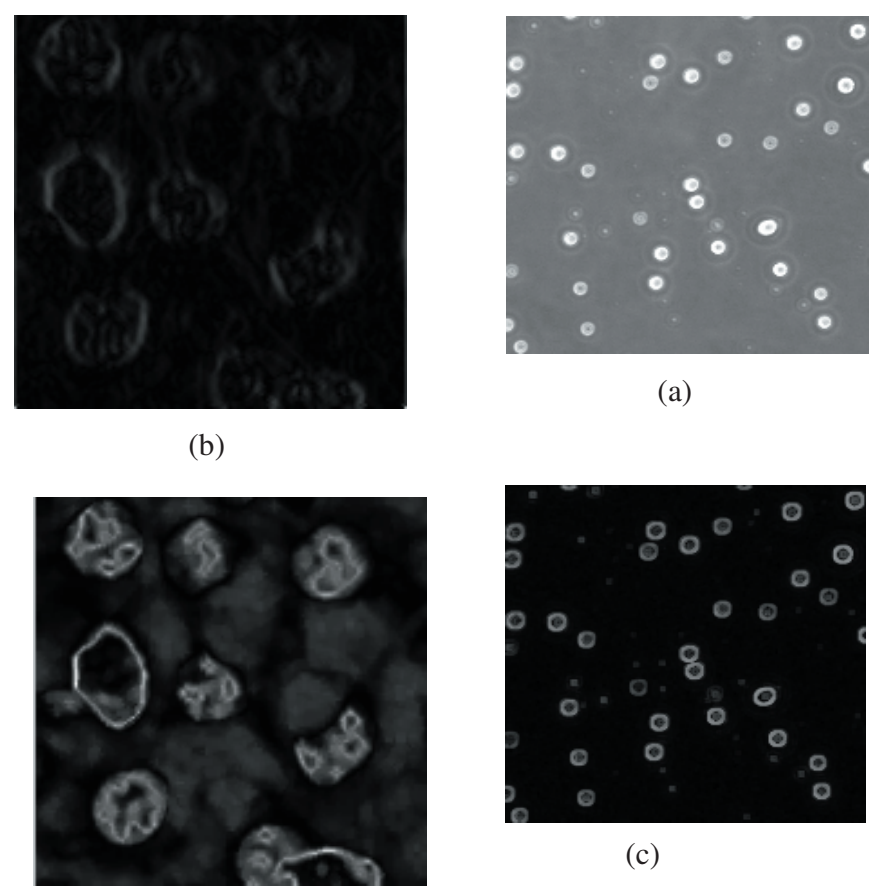

(c)

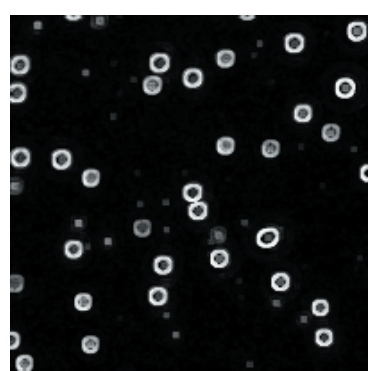

(e)

Fig. 7. : a) blood cell image, b) edge image using fuzzy morphology, c) edge image using the interval valued fuzzy set, d) edge image by Chaira and Ray, e) edge image using proposed method 


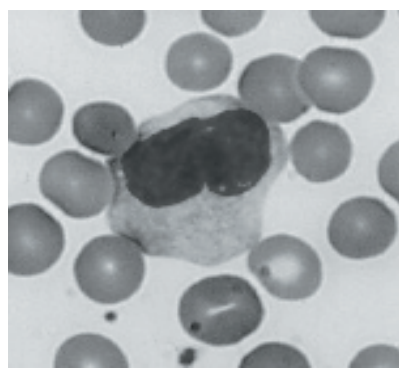

(a)

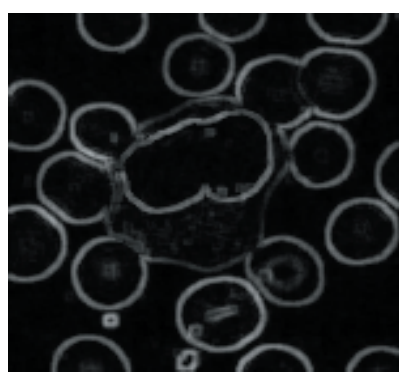

(c)

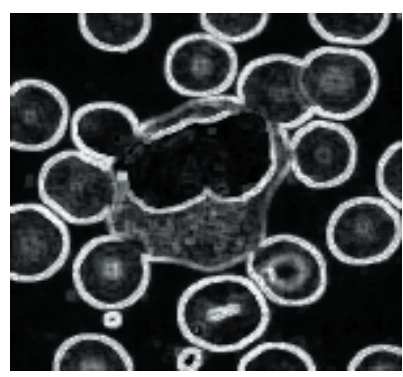

(e)

Fig. 8 : a) blood cell image, b) edge image using fuzzy morphology, c) edge image using the interval valued fuzzy set, d) edge image by Chaira and Ray, e) edge image using proposed method

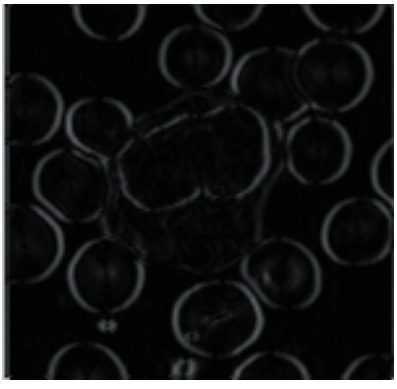

(b)

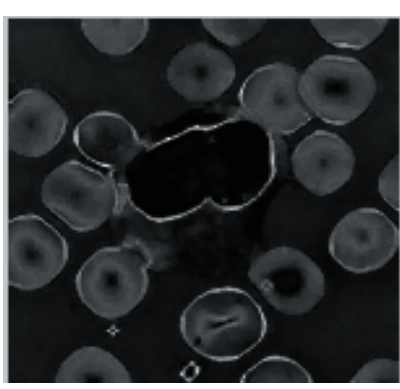

(d)

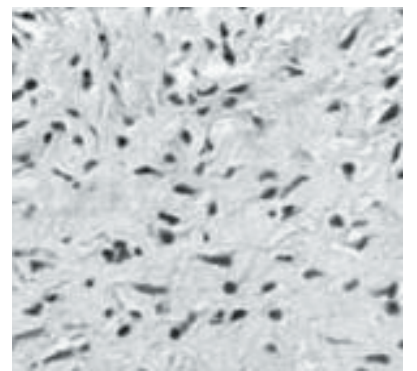

(a)

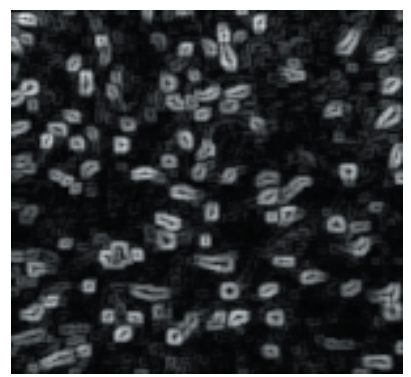

(c)

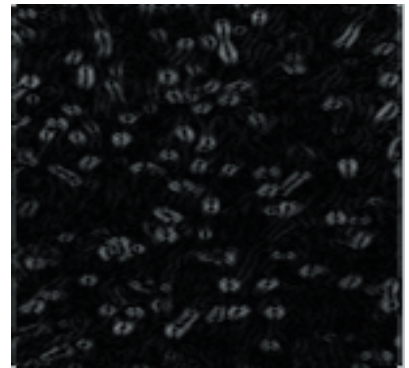

(b)

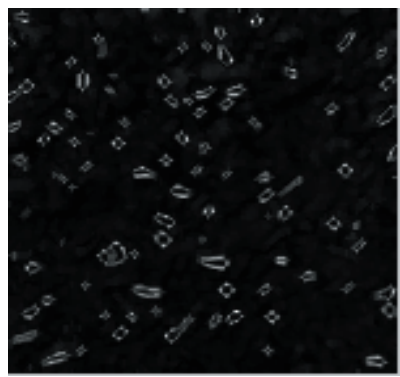

(d)

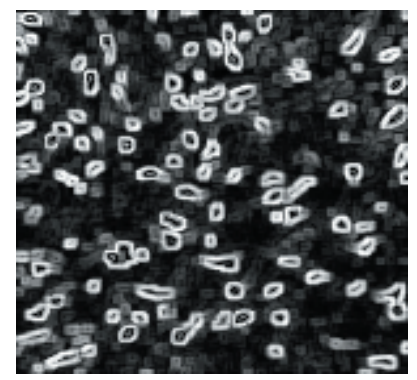

(e)

Fig. 9 : a) nuclei image, b) edge image using fuzzy morphology, c) edge image using the interval valued fuzzy set, d) edge image by Chaira and Ray, e) edge image using proposed method 


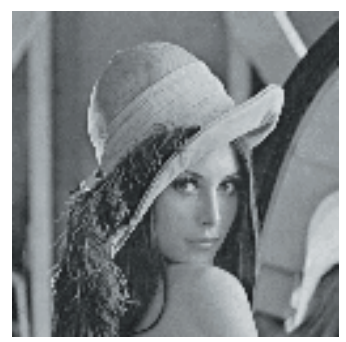

(a)

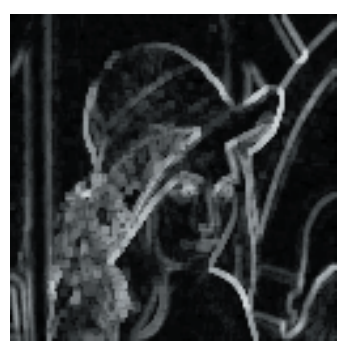

(c)

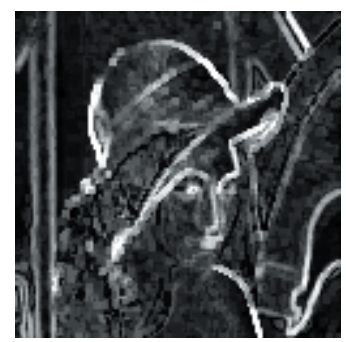

(e)

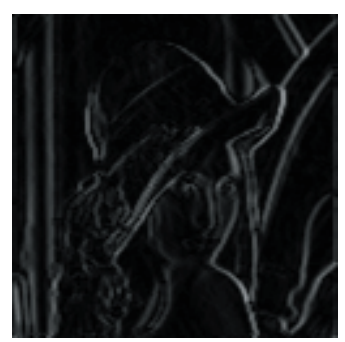

(b)

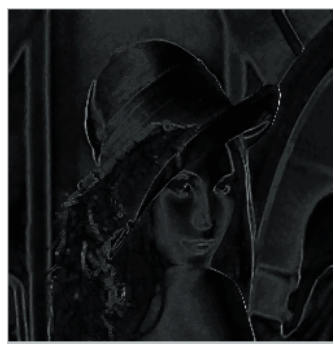

(d)
Fig. 10. : a) Lena image, b) edge image using fuzzy morphology, c) edge image using the interval valued fuzzy set, d) edge image by Chaira and Ray, e) edge image using proposed method

\section{Conclusion}

An idea to construct fuzzy edge image of medical images using Type II fuzzy set theory is suggested. The method uses upper and lower membership levels of the maximum and the minimum valued image matrices that are formed in a $3 \times 3$ pixel neighborhood using Type II fuzzy set. Fuzzy divergence is computed between two levels for the maximum value image matrix and also for the minimum value image matrix. Fuzzy edge image is obtained by taking the difference of the two divergence matrices.

In this method, the minimum and maximum valued matrices are not considered precise and so for better representation of uncertainty, their upper and lower membership levels using Type II fuzzy set are computed. Then the difference of the two divergences is the edge image. This is the reason why the edges of the proposed method are bright.

It is useful to those images where the edges are not visible properly i.e. the images which are poorly illuminated. The resulting edges look very bright using the proposed method. One can have clear picture about the presence of edges. Experimental results show that the algorithm is efficient on medical images.

When the membership function is not accurately defined, Type II fuzzy set is very helpful that takes the interval range of the membership function. This may be the reason that the proposed method is much better than the existing methods.

\section{Acknowledgement}

Author would like to acknowledge the anonymous reviewers for their valuable comments in improving the quality of the manuscript.

Authors would also like to acknowledge the Department of Biotechnology, Govt. of India for financially supporting the research work carried out under the National award scheme 'Innovative Young Biotechnologist Award, IYBA-2010”.

\section{References}

1. L.A. Zadeh, Fuzzy Sets, Information and Control 8 (1965) 338-353.

2. L.A. Zadeh, The Concept of a Linguistic Variable and Its Application to Approximate Reasoning-1, Information Sciences 8 (1975) 199-249.

3. H.R. Tizhoosh, Image thresholding using type II fuzzy sets, Pattern Recognition 38(2) (2005) 2363-2372.

4. H. Bustince, E. Barrenechea, M. Pagola, J. Fernandez, J. Sanz, Image thresholding using type II fuzzy sets". Importance of this method, Pattern Recognition 43(9) (2010) 3188-3192.

5. R.C. Gonzalez and W.E. Woods, Digital image processing $2^{\text {nd }}$ edition, Prentice Hall, Upper Saddle River, NJ.

6. J. Canny, A computational approach to edge detection, IEEE Transaction on Pattern Analysis Machine Intelligence 8(6) (1986) 679-698.

7. F. Russo, FIRE operators for image processing, Fuzzy Sets and Systems. 10 (1999) 265-275.

8. F. Russo and G. Rampone, Edge detection by FIRE operators, in Proc. of IEEE Conferance on Fuzzy systems (1994) 249-253.

9. L.R. Liang and C.G. Looney, Competitive fuzzy edge detection, Applied Soft Computing 3, 123-137, 2003.

10. T. Chaira and A.K. Ray, A new measure on intuitionistic fuzzy set and its application to edge detection, Applied soft computing 8(2) (2008) 919-927. 
11. E. Barrenchea, et. al, Construction of interval valued fuzzy relation with application to generation of fuzzy edge images, IEEE Transaction on Fuzzy Systems 9 (5) (2011).

12. H. Bustince, E. Barrenechea, M., Pagola, J. and Fernandez, Interval valued fuzzy sets constructed from matrices: Application to edge detection, Fuzzy Sets Systems 160 (2009), 1819-1840.

13. A.PAHSA, Morphological image processing using fuzzy logic, HAVACILIK VE UZAY TEKNOLOJILERİ DERGISİ, OCAK (2006) 27-34.

14. J. Mende and B. John, Type 2 Fuzzy sets made simple, IEEE Transaction on Fuzzy Systems 10(2) (2002) 117127.

15. J.M. Mendel, R.I. John and F. Liu, Interval type-2 fuzzy logic systems made simple, IEEE Trans. Fuzzy Systems 14(6) (2006) 808-821.

16. P. Ensafi and H.R. Tizoosh, Type II fuzzy image enhancement, in Lecture Notes in Computer Sciences, vol. 3656, in M. Kamel and A. Campilho, Ed.:Springer (2005) 159-166. 